\title{
TWO SAMPLE SPUTUM MICROSCOPY AND REVISED TUBERCULOSIS CASE DEFINITION BY WHO : BANGLADESH PERSPECTIVE
}

Dewan $G^{1}$

\begin{abstract}
INTRODUCTION: Three samples of sputum (Spot-Morning-Spot) used for screening patients with suspected pulmonary tuberculosis in Bangladesh. At least two positive samples required for case definition. Cases with single positive smear require additional chest $\mathrm{x}$-ray or culture. The study was done to evaluate utility of two sputum samples for case detection and whether adoption of new case definition (requiring single positive sample) recommended by World Health Organization adds advantage over current case definition for diagnosing smear positive pulmonary tuberculosis.
\end{abstract}

MATERIAL AND METHODS: Retrospective analysis of sputum positive pulmonary tuberculosis cases taken from two separate hospitals in southeast Bangladesh. Frequency, pattern and increment of smear positivity noted in each samples. Smear positive cases reevaluated using proposed case definition and compared with current national definition.

RESULTS: Sputum positivity for first, second and third samples was 71.6\%, 99.0\% and 97.3\% respectively ( $\mathrm{n}=408$ ). Incremental new case detection rate was $71.6 \% .28 .1 \%$ and $0.2 \%$ in same order. Morning sputum had the highest sensitivity for case detection (99.0\%). Incremental new case detection from third sample was negligible and using current case definition of sputum positive tuberculosis in three samples left some inconclusive cases requiring further $\mathrm{x}-$ ray or culture for diagnosis pending decision further. Adoption of new case definition reduces number of inconclusive cases and diagnostic delay.

CONCLUSION: Two sputum samples adequate for screening of tuberculosis suspects. Adopting new case definition will reduce number of cases left inconclusive with microscopy alone (using current case definition). This will strengthen role of microscopy in tuberculosis detection in low resource setting.

KEYWORDS: Tuberculosis, Sputum, Case definition, Bangladesh

1. Consultant, Department of Medicine, Rangamati General Hospital, Rangamati, Bangladesh

\author{
For Correspondence \\ Dr. Gourab Dewan \\ Consultant, \\ Department of Medicine, \\ Rangamati General Hospital, Rangamati, Bangladesh \\ E-mail: gourab.dewan@yahoo.com
}




\section{INTRODUCTION}

For low resource setting countries sputum microscopy is the standard for detecting pulmonary tuberculosis (PTB). It has limitations as well. For long time the ideal number of sputum sample required for detection of tuberculosis has been a matter of debate. World Health Organization (WHO) has recommended both two as well as three sample study of sputum depending on presence or absence of external quality assessment (EQA) system or local policy (ie countries not willing to introduce two sample study despite well-developed EQA system). ${ }^{1}$ Traditionally two positive samples required for case definition of PTB but recently WHO revised case definition for countries with well developed EQA where single positive sample is enough. ${ }^{2}$

Bangladesh is sixth among countries with high TB burden. ${ }^{3}$ Though EQA is well developed the strategy still based on 3 spot-morning-spot (S-M-S) sputum samples to detect open PTB. Definite diagnosis of PTB with microscopy alone requires at least two positive samples. ${ }^{4}$ A single positive smear requires either a compatible lesion in chest X-ray or positive yield in culture for definite diagnosis as PTB. The questions are; has time come to change detection strategy towards two sample approach in Bangladesh? And is there any advantage if WHO's revised case definition of single positive sputum sample adopted along with two sample screening?

In 2002 in a study among Bangladeshi population Van Deun et al. after comparing different combination of sputum collection suggested two samples both collected in morning was adequate. ${ }^{5}$ In 2013 a study done among prisoners (a highly risk group) in Bangladesh again suggests two sample may be adequate. But the statement of authors was cautious; calling for further study in different setting before generalization of their result. ${ }^{3}$ Therefore it was decided to evaluate the reproducibility of the findings of the mentioned study in general population. If two sample screening proves effective it will reduce work load as well as cost- a vital issue for developing countries.

\section{MATERIAL AND METHODS}

A retrospective cross sectional study was done in chest disease clinic (CDC) of Rangamati and Rangamati General Hospital (RGH). Rangamati is the largest district of Bangladesh lying on southeastern part. Sputum examination records were reevaluated from both centres. In CDC data was available from 2005 to 2013 while in RGH from 2008 to 2013. Only those persons who underwent sputum test for diagnosis of tuberculosis were included. Follow up cases and incompletely submitted samples excluded. Sputum was collected spotmorning-spot basis. Frequency of sputum positivity with each sample noted. Incremental TB detection rate with 1st, 2nd and 3rd sample were compared. Any level of significance was tested with chi-square test and Fisher's exact test as applicable. Level of significance defined as $\mathrm{p} \leq 0.05$ using $95 \%$ confidence interval. SPSS version 20 used for data processing

Currently national policy follows WHO's recommendation for countries with less established EQA of at least two smear positive sample out of three for definite diagnosis as PTB. All cases were reevaluated using WHO's revised case definition (in first two samples only). First, second and third samples designated as $\mathrm{S} 1, \mathrm{~S} 2$ and $\mathrm{S} 3$ respectively.

\section{RESULT}

A total of 9228 person underwent sputum microscopy in CDC and in RGH within the specified period. With predetermined inclusion criteria final recruitment for study was 408 in number. Frequency of acid fast bacilli (AFB) detection in each samples presented in Table 1. First sample had the lowest case detection rate (71.6\%). Overall positivity rate of S2 $(99.0 \%)$ and S3 $(97.3 \%)$ are almost identical. Compared to S1 both S2 and S3 showed increased case detection rate of $27.4 \%$ $(\mathrm{p}<0.0001)$ and $25.7 \%(\mathrm{p}<0.0001)$ respectively.

\section{Table 1: Frequency of sputum positivity in each sample}

\begin{tabular}{|l|c|c|c|c|c|c|}
\hline \multirow{2}{*}{$\begin{array}{c}\text { Result of } \\
\text { sputum } \\
\text { examination }\end{array}$} & \multicolumn{2}{|c|}{$1^{\text {st }}$ sample } & \multicolumn{2}{c|}{$2^{\text {nd }}$ sample } & \multicolumn{2}{c|}{$3^{\text {rd }}$ sample } \\
\cline { 2 - 7 } & Number & $\%$ & Number & $\%$ & Number & $\%$ \\
\hline Positive & 292 & 71.6 & 404 & 99.0 & 397 & 97.3 \\
\hline Negative & 116 & 28.3 & 4 & 0.9 & 11 & 2.6 \\
\hline
\end{tabular}

Incremental new case detection with $\mathrm{S} 1$ and $\mathrm{S} 2$ was $71.6 \%$ and $28.1 \%$ respectively. Combined first two samples detected $99.7 \%$ of total cases. New case detection yield of third sample was only $0.2 \%$ (Table 2 ).

Table 2: Incremental new case detection in successive sputum samples

\begin{tabular}{|l|c|c|}
\hline Sputum sample & Case detected & Percentage increment \\
\hline First sample (Spot) & 292 & 71.6 \\
\hline Second sample (Morning) & 115 & 28.1 \\
\hline Third sample (Spot) & 1 & 0.2 \\
\hline
\end{tabular}

Majority (69.1\%) were positive in all samples. Two samples 
were positive in $29.6 \%$. Single sample was positive in $1.2 \%$ (Table 3).

Table 3: Pattern of sputum positivity for AFB in three samples

\begin{tabular}{|l|c|c|}
\hline Pattern of smear positivity & Number & Percentage \\
\hline All three positive & 282 & 69.1 \\
\hline S1 and S2 positive & 7 & 1.7 \\
\hline S2 and S3 positive & 113 & 27.7 \\
\hline S1 and S3 positive & 1 & 0.2 \\
\hline Only S1 positive & 2 & 0.5 \\
\hline Only S2 positive & 2 & 0.5 \\
\hline Only S3 positive & 1 & 0.2 \\
\hline
\end{tabular}

* S1= First spot specimen, $\mathrm{S} 2=$ Morning specimen,

$\mathrm{S} 3=$ Second spot specimen

Among 115 cases when S2 was the first positive sample S3 also positive in $113(98.2 \%)$ of them. Considering combination of any two samples; $\mathrm{S} 1+\mathrm{S} 2$ new case detection rate was $99.7 \%(\mathrm{n}=407)$ while $\mathrm{S} 2+\mathrm{S} 3 / \mathrm{S} 1+\mathrm{S} 3$ new case detection rate was $99.5 \%(\mathrm{n}=406)$.

With current national case definition 403 (98.7\%) were marked as definite PTB case (Table 4).

Table 4: Comparison of WHO's recommended two and three sample sputum collection methods \& case definitions $(\mathrm{N}=408)$

\begin{tabular}{|l|c|c|c|c|}
\hline & \multicolumn{2}{|c|}{ Current three sample method } & \multicolumn{2}{c|}{$\begin{array}{c}\text { Proposed two } \\
\text { sample method }\end{array}$} \\
\cline { 2 - 5 } & $\mathrm{N}$ & $\%$ & $\mathrm{~N}$ & $\%$ \\
\hline Case confirmation with S1 & ---- & ---- & 292 & $71.5 \%$ \\
\hline Case confirmation after S2 & 289 & $71.7 \%$ & 115 & $28.1 \%$ \\
\hline Case confirmation after S3 & 114 & $28.2 \%$ & --- & --- \\
\hline Inconclusive case & 5 & $1.2 \%$ & --- & $\cdots$ \\
\hline Negative case & ---- & --- & 1 & $0.2 \%$ \\
\hline
\end{tabular}

* At least two sample positive for case confirmation

${ }^{+}$Single positive sample satisfies case definition

Sensitivity of S1, S2 and S3 in detecting definite 403 PTB cases were $71.9 \%(n=290), 99.7 \%(n=402)$ and $98.2 \%$ $(n=396)$ respectively. The observed difference in sensitivity of
S2 with S3 $(\mathrm{p}<0.03)$ and S1 $(\mathrm{p}<0.0001)$ was statistically significant. Positive predictive value (PPV) of S2 for a definite PTB case was $99.7 \%$. And that of S3 and S1 was $98.2 \%$ and $71.9 \%$ respectively. Five cases $(1.2 \%)$ remained inconclusive. When only S1 and S2 were reevaluated using revised case definition 407 cases $(99.7 \%)$ were marked as definite PTB $(\mathrm{S} 1=292$ and $\mathrm{S} 2=115)$ and one case was negative. Importantly no case was left inconclusive. The only new case detected with $\mathrm{S} 3$ was missed with revised definition as $\mathrm{S} 3$ was not examined (Table 5). The difference in number of inconclusive cases left after microscopy alone by two different screening method was statistically significant $(\mathrm{p}<0.03)$.

Table 5: Comparison of incremental yield in AFB detection with successive sputum samples in studies of Bangladesh

\begin{tabular}{|l|c|c|c|c|c|}
\hline \multicolumn{1}{|c|}{ Studies } & Sample collection & Cases & $\begin{array}{c}\text { S1 } \\
\mathbf{( \% )}\end{array}$ & $\begin{array}{c}\text { S2 } \\
\mathbf{( \% )}\end{array}$ & $\begin{array}{c}\text { S3 } \\
\mathbf{( \% )}\end{array}$ \\
\hline Rangamati (2014) & S-M-S & 408 & 71.6 & 28.1 & 0.2 \\
\hline Islam et al. (2013) & S-M-M & 334 & 89 & 9 & 2 \\
\hline $\begin{array}{l}\text { Van Deun et al. } \\
(2002)^{5}\end{array}$ & S-M-S & 4865 & 82.5 & 15.2 & 2.3 \\
\cline { 2 - 6 } & S-M-M & 4774 & 79.8 & 17.9 & 2.3 \\
\cline { 2 - 7 } & M-M-M & 3077 & 94.2 & 4.8 & 1 \\
\hline
\end{tabular}

* $\mathrm{S}=$ Spot, $\mathrm{M}=$ Morning

\section{DISCUSSION}

This retrospective study confirms that in setting of Bangladesh adoption of two sample sputum screening strategy and single positive sample for definite diagnosis of PTB (as per WHO recommendation for countries with wellestablished EQA system) will improve detection of definite PTB cases using microscopy alone and reduce number of inconclusive cases requiring further X-ray or culture with current three sample screening. The method will reduce workload of manpower concerned and in turn expected to improve case detection efficiency as lab technicians can devote more time for examining individual slides.

The rationale behind three sample sputum examination is that some patient shed tubercular bacilli irregularly therefore increase in sample number increases chance of detection. ${ }^{6}$ More over a morning samples gives highest microbiological yield. Current finding of $99.7 \%$ detection rate with first two samples is in accordance with prior studies. Mase et al. in his review of 37 studies observed cumulative incremental detection rate with first two sample to be $97.7 \%{ }^{7}$. Yield with $3^{\text {rd }}$ sample is almost negligible $(0.2 \%)$ in current series. It will require examining about 500 slides to detect an additional new 
case from S3. Cumulative yield of S3 in review of Mase et al. was $3.1 \%$, while Rieder et al. observed a range of 0.7 to $7.2 \%$ by reviewing 42 studies. ${ }^{7,8}$

Finding of current study compared with prior two studies among Bangladeshi population in Table 5. All studies agrees that yield of third sputum is negligible ( 0.2 to $2.3 \%)$ whatever may be the timing of collection. Current yield of $0.2 \%$ with S3 is lowest among three native studies.

Second spot sample in Rangamati had higher case detection rate than first spot sample $(97.3 \%$ vs $71.6 \%)$. But morning sputum (S2) had highest yield (99.0\%). Our yield in S2 is highest among all Bangladeshi studies. It again reconfirms the value of morning sputum in detecting AFB. Our case detection rate with $\mathrm{S} 1$ is lowest among Bangladeshi studies. Compared to nearby countries; this value is much lower than studies by Rao et al. (89.1\%), Hamid et al. (91.8\%) in India and Wu et al. $(84.5 \%)$ in China. ${ }^{9-11}$ However this value higher than Saleem et al. $(66.2 \%)$ in Pakistan. ${ }^{12}$

If sole reliance placed on morning sample it almost detected all smear positive cases in this series (sensitivity and PPV 99.7\%). As no culture was done we could not calculate specificity in this series. However in high endemic countries microscopy may be more specific than culture. Several studies found that new case detection rate with $\mathrm{S} 1+\mathrm{S} 2$ is almost equal to new case detection rate with culture of first sample of sputum. $^{13}$

By S1+S2 using national case definition 70.8\% (289) suspects were labeled as definite PTB case so carrying out S3 for those cases was unnecessary. Each slide preparation and examination takes 30 minutes to 3 hours depending on bacilli load. ${ }^{14}$ Elimination of S3 from them would have cut down work load by $23.8 \%$. No attempt made here to calculate the cost that could have been saved in terms of reagent. $\mathrm{S} 2+\mathrm{S} 3$ confirmed further $113(27.7 \%)$ and $\mathrm{S} 1+\mathrm{S} 3$ confirmed $1(0.2 \%)$ case. Mase et al. observed reduction of smear number from three to two does not significantly reduce overall case detection rate but reduces cost and saves time of lab personnel's significantly.?

Should we need S3? Sensitivity and PPV of S2 very high (99.7\%) for definite PTB cases; when S2 is the first positive sample the need for $\mathrm{S} 3$ is also questionable as concurrent $\mathrm{S} 3$ positivity in such situation almost identical (98.2\%). As mentioned; a new case detected by S3 after $\approx 500$ additional slides checking in Rangamati. Even then such single new positive sample with $\mathrm{S} 3$ requires categorization as inconclusive and will require $\mathrm{x}$-ray/ culture to level as definite case as per national policy. Therefore utility of S3 for new case detection is a matter of question. Only benefit of $3^{\text {rd }}$ sample was microscopic confirmation of smear positive TB by demonstrating AFB in two samples (S1/S2 +S3) and thereby excluding chance of false positive /contamination in $27.9 \%$ cases $(n=114)$.Considering three stage quality assessment running in country we have to place confidence on our system and manpower. More over elimination of S3 may enhance case detection rate as technician can spent more time on samples with low bacilli load. However in neighboring Pakistan a study found result in favor of three samples as yield with $\mathrm{S} 3$ was $9.8 \%(n=43)^{9}$.

Now comparison of results using current national case definition and WHO's revised case definition discussed (Table $4)$. With current policy five $(1.2 \%)$ cases remained inconclusive after three sample test. It is proven in such cases further sputum exam is of little value. ${ }^{13}$ Rao et al. in India had $9 \%(n-50)$ of cases in his series positive in only single sample and required X-ray. ${ }^{6}$ A very high inconclusive cases of $41.3 \%$ $(n=181)$ obtained by Saleem et al. in Pakistan. ${ }^{9}$ Currently for inconclusive cases coverage of expenses for any travel and $\mathrm{x}-$ ray/ culture being offered to patients in Bangladesh. If revised case definition by WHO applied; $99.7 \%$ cases $(n=407)$ labeled as definite PTB by two smears. It missed the sole newly detected case with S3. Moreover those cases labeled inconclusive with three sample screening now labeled as definite PTB case not requiring X-ray or culture. So it is cost effective. Importantly almost identical number of cases (289 Vs 292) were identified as definite PTB with second visit in current national policy and with first visit in revised definition. It shows revised definition cuts down delay in diagnostic confirmation and decision to treat by a day in $71.5 \%(n=292)$. If two smear to be adopted question arises which two samples to be used? $\mathrm{S} 1+\mathrm{S} 2$ or $\mathrm{S} 1+\mathrm{S} 3 / \mathrm{S} 1+\mathrm{S} 3$ ? There s almost identical new case detection rate among these combinations. However here recommendation made for $\mathrm{S} 1+\mathrm{S} 2(\mathrm{~S}-\mathrm{M})$ as because $\mathrm{S} 2$ gives the highest yield and improvement in coughing technique of the patient by the time $\mathrm{S} 2$ submitted has some role to play in increasing yield besides being morning sample which traditionally considered to have high AFB. This observation of current study reconfirms the recommendation that in highly endemic countries reduction of smears to two including at least one morning sample may be adequate. ${ }^{15-17}$

\section{CONCLUSIONS}

In Bangladesh majority cases of open PTB can be confidently identified with two sample sputum (spot-morning). Current finding is in agreement with prior studies in Bangladesh (though difference in sample collection exists) that new case 
detection with third sputum is negligible. Number of inconclusive cases seen with current diagnostic criteria can be reduced applying WHO's revised case definition. Both strategies will be cost effective, reduce laboratory burden and quicken case management.

\section{REFERENCES}

1. World Health Organization. Proposed reduction of number of smears for the diagnosis of pulmonary TB: background document. [Internet] 2009 [cited 28 January 2014]. Available from: http://www.who.int/tb/laboratory/reduction_of_smears. $p d f$

2. World Health Organization. Treatment of tuberculosis guidelines. 4thed. Geneva: WHO; 2010

3. Islam MR, Khatun R, Uddin MKM, Khan MSR, Rahman M, et al. (2013) Yield of Two Consecutive Sputum Specimens for the Effective Diagnosis of Pulmonary Tuberculosis. PLoS ONE 8(7): e67678. doi:10.1371/journal.pone.0067678 http://dx.doi.org/ 10.1371/journal.pone.0067678

4. National Tuberculosis Control Programme (NTP): Achieved and Yet to Achieve (Editorial). Birdem Med J 2013; 3(1): 1-3

5. Van Deun A, Salim AH, Cooreman E, Hossain MA, Rema A, Chambugonj Net al. Optimal tuberculosis case detection by direct sputum smear microscopy: how much better is more? Int $J$ Tuberc Lung Dis 2002; 6(3):222-30. PMid:11934140

6. Mathew P, Kuo Y, Vazirani B, Eng RHK, Weinstein MP. Are Three Sputum Acid-Fast Bacillus Smears Necessary for Discontinuing Tuberculosis Isolation? J Clin Microbiol 2002; 40 (9) : 3482-84. PMid:12202598 PMCid:PMC130719

7. Mase S, Ramsay A, $\operatorname{Ng~N,~Henry~M,~Hopewell~PC,~Cunningham~}$ $J$ et al. Yield of serial sputum specimen examinations in the diagnosis of pulmonary tuberculosis: a systematic review. Int $J$ Tuberc Lung Dis 2007; 11 (5): 485-495. PMid:17439669

8. Rieder HL, Chiang CY, Rusen ID. A method to determine the utility of the third diagnostic and the second follow-up sputum smear examinations to diagnose tuberculosis cases and failures. Int J Tuberc Lung Dis 2005; 9 (4): 384-391.PMid:15830743

9. Rao S. Sputum smear microscopy in DOTS: Are three samples necessary? An analysis and its implications in tuberculosis control. Lung India 2009; 26(1): 3-4. Http://dx.doi.org/ 10.4103/0970-2113.45196 PMid:20165586 PMCid:PMC 2813116

10. Hamid S, Hussain SA, Imtiyaz A. Screening tuberculosis suspects: How many sputum specimens are adequate?. Ann Trop Med Public Health 2012; 5:317-20. Http://dx.doi.org/ 10.4103/1755-6783.102035

11. Wu ZL, Wang AQ. Diagnostic yield of repeated smear microscopy examinations among patients suspected of pulmonary TB in Shandong province of China. Int $J$ Tuberc Lung Dis 2000; 4(11):1086-87. PMid:11092724

12. Saleem S, Shabbir I, Iqbal R, Khan SU. Value of Three Sputum Smears Microscopy in Diagnosis of Pulmonary Tuberculosis. PakJMed Res. 2007; 46 (4): 94-7.

13. Frieden T (ed). Toman's tuberculosis case detection, treatment, and monitoring. 2nded. Geneva : WHO; 2004

14. Sotaquirá M, Rueda L, Narvaez R. Detection and quantification of bacilli and clusters present in sputum smear samples: a novel algorithm for pulmonary tuberculosis, proceedings of international conference on digital image processing. [Internet] 2009 [cited 28 January 2014]. Available from: http://cdn.intechopen.com/pdfs-wm/28543.pdf

15. Sarin R, Mukerjee S, Singla N, Sharma PP. Diagnosis of tuberculosis under RNTCP: examination of two or three sputum specimens. Indian J Tuberc 2001; 48: 13-6.

16. Walker D, McNerney R, Kimankinda M, Foster, S.; Tihon, V.; Godfrey-Faussett, $P$ et al. An incremental cost-effectiveness analysis of the first, second and third sputum examination in the diagnosis of pulmonary tuberculosis Int J Tuberc Lung Dis 2000; 4: 246-51. PMid:10751071

17. Mabaera B, Naranbat N, Dhliwayo P, Rieder H L. Efficiency of serial smear examinations in excluding sputum smear-positive tuberculosis. Int J Tuberc Lung Dis 2006; 10: 10301035. PMid:16964796 